ORIGINAL ARTICLE

\title{
Physician variability in treating pain and irritability of unknown origin in children with severe neurological impairment
}

\author{
Harold B Siden MD MHSc${ }^{1}$, Bruce C Carleton PharmD², Tim F Oberlander MD
}

\begin{abstract}
HB Siden, BC Carleton, TF Oberlander. Physician variability in treating pain and irritability of unknown origin in children with severe neurological impairment. Pain Res Manag 2013;18(5):243-248.
\end{abstract}

BACKGROUND: Pain and irritability of unknown origin (PIUO) is a challenging problem for nonverbal children with severe neurological impairments. PIUO is not associated with an identifiable source of nociceptive-inflammatory or neuropathic pain.

OBJECTIVE: To assess how physicians use pharmacotherapy to treat PIUO, and to report a pilot study of a standardized approach to investigating and treating PIUO.

METHOD: Part 1 of the present study involved independently presenting a case vignette of a patient with PIUO to six experienced physicians who care for children with neurological impairments. They were asked for medication choices and sequences to empirically treat PIUO. Part 2 was a pilot study of a PIUO protocol. Patients followed a standard pathway for PIUO, referred to as the pathway for unknown pain (PUP). The initial drug sequence for the PUP was based on Part 1.

RESULTS: In Part 1, physicians responding to the case vignette listed eight medications (atypical antipsychotics, benzodiazepines, gabapentin, methadone, opioids, selective serotonin reuptake inhibitors, tramadol and tricylic antidepressants) and eight empiric drug sequences. In Part 2, eight children with PIUO (six to 17 years of age; five females, three males) were enrolled in a pilot clinic. Only two had been fully evaluated for nociceptive-inflammatory pain sources before enrollment. At the end of the pilot study, four patients were clinically improved and only three required a study medication.

DISCUSSION AND CONCLUSION: Even experienced physicians do not agree on a common approach for medical treatment of PIUO. A standardized pathway is feasible and readily implemented. The proposed PUP has the potential to address PIUO and be the basis for future intervention studies.

Key Words: Decision making; Developmental disability; Pain management; Pediatric pain

\author{
La variabilité des médecins dans le traitement de la \\ douleur et de l'irritabilité d'origine inconnue chez \\ des enfants présentant des atteintes neurologiques \\ graves
}

HISTORIQUE : La douleur et l'irritabilité d'origine inconnue (DIOI) sont un problème complexe pour les enfants non verbaux ayant des atteintes neurologiques graves. Les DIOI ne s'associent pas à une source identifiable de douleurs inflammatoires nociceptives ou neuropathiques.

OBJECTIF : Évaluer la manière dont les médecins utilisent la pharmacothérapie pour traiter les DIOI et rendre compte du projet pilote d'une démarche standardisée pour examiner et traiter les DIOI.

MÉTHODOLOGIE : La première partie de la présente étude portait sur la présentation indépendante de l'étude de cas d'un patient ayant des DIOI à six médecins expérimentés qui soignent des enfants ayant des atteintes neurologiques. On leur a demandé leurs choix pharmacologiques et leurs séquences de traitement empirique des DIOI. La deuxième partie était le projet pilote d'un protocole de DIOI. Les patients suivaient une voie standard de DIOI, désignée comme la voie de la douleur inconnue (VDI). La première séquence pharmacologique de la VDI se fondait sur la première partie.

RÉSULTATS : Pendant la première partie, les médecins qui ont répondu à l'étude de cas ont dressé une liste de huit médicaments (antipsychotiques atypiques, benzodiazépines, gabapentine, méthadone, opiö̈des, inhibiteurs sélectifs du recaptage de la sérotonine, tramadol et antidépresseurs tricycliques) et huit séquences pharmacologiques empiriques. Dans la deuxième partie, huit enfants ayant des DIOI (six à 17 ans; cinq filles, trois garçons) ont participé à une clinique pilote. Seulement deux ont subi une évaluation complète des sources de douleurs inflammatoires nociceptives avant leur participation à la clinique. À la fin du projet pilote, l'état de quatre patients s'était amélioré sur le plan clinique, et seulement trois ont eu besoin d'un médicament à l'étude.

EXPOSÉ ET CONCLUSION : Même des médecins expérimentés ne s'entendent pas sur une approche commune pour le traitement pharmacologique des DIOI. Une voie standardisée est faisable et facile à mettre en œuvre. La VDI proposée a le potentiel de soulager les DIOI et de jeter les bases de futures études d'intervention. individual would be insensitive or indifferent to pain. There is little agreement on the best approach to unexplained irritability in children with SNI.

Pain-related behaviours have been recognized as a frequent characteristic of daily life in children with SNI (2-8). Painful experiences arise from multiple sources associated with injury, inflammation and nociception $(9,10)$. These children undergo repetitive invasive medical and surgical procedures, each contributing to pain. In addition, pain can result from musculoskeletal problems, infections, gastrointestinal dysfunction, pressure sores or irritated gastrostomy sites. Such known sources of pain can typically be identified through thorough history-taking, physical examination, laboratory tests and imaging studies, leading to appropriate treatment using standard analgesics that address nociceptive-inflammatory pain (11). the life of someone with a developmental disability or that such an

${ }^{1}$ Division of General Pediatrics, Canuck Place Children's Hospice; ${ }^{2}$ Division of Translational Therapeutics; ${ }^{3}$ Division of Developmental Pediatrics, Department of Pediatrics, University of British Columbia, Vancouver, British Columbia

Correspondence: Dr Harold B Siden, 4500 Oak Street, Vancouver, British Columbia V6H 3N1. Telephone 604-875-2776, fax 604-875-3284, e-mail hsiden@cw.bc.ca 
In many cases, however, even with a thorough examination, a correctable source of pain cannot be identified. In the absence of an identified nociceptive-inflammatory source, the ambiguous nature of 'pain-like' behaviours, such as facial grimacing, crying/vocalizations, posturing and limb movement, leads to diagnostic and treatment uncertainty (5). Management then shifts from treating inflammatorynociceptive pain to using an empirical approach to address pain-like behaviours assumed to reflect a neuropathic and central nervous system pain signal $(9,12)$. There are no guidelines to direct a standard approach in this situation. Empirical approaches rely on assumptions that particular neurotransmitters mediate the irritability, leading to use of medications that commonly include anti-inflammatory drugs (eg, nonsteroidal anti-inflammatory drugs, acetaminophen), tricyclic antidepressants, neurotransmitter reuptake inhibitors (eg, fluoxetine), anticonvulsants (eg, gabapentin, carbamazepine), antispasmodic agents (eg, baclofen, benzodiazepines), antipsychotics (eg, risperidone) and opioids.

Our limited understanding of the exact nature of persistent pain and irritability in children with SNI is a major barrier to treatment. The constellation of findings may be described using a variety of terms such as pain-like behaviour, pain/irritability, central irritability, neuropathic pain, central neuropathic pain or neuroirritability. For the purposes of the present article, this entity will be referred to as pain/ irritability of unknown origin (PIUO). To date, little is known about whether biochemical, physiological or structural neurological changes associated with an SNI contribute to our understanding of the pain experience or how to develop a systematic approach to treatment. The sequence or nature of the medications used differs from one physician to another, and often without a specific rationale. (Siden H, Hain R, Friedrichsdorf S, Gilles S, Hauer J, personal communication). Recently, gabapentin has been considered to be a first-line therapy for PIUO; however, the evidence for this recommendation is limited, based on expert opinion articles and one case series of seven patients (13). To date, there are no studies to guide the sequence of medication trials beyond gabapentin. Small cohorts, heterogenic conditions and unpredictable pain patterns render typical research designs, such as case-cohort and randomized controlled trials, inadequate to address questions about how to assess pain and determine analgesic efficacy in this setting. New approaches are needed that offer the capacity for a rapid response as well as the flexibility to address individual needs.

To address the need for a systematic approach to PIUO management in children with SNI, we undertook the present study to evaluate the consistency of the physician-directed drug therapeutic approach (study 1) and, using these findings, undertake a pilot study to apply a systematic approach to managing PIUO (study 2 ). In study 1 , we identified the pharmacotherapy approaches used by a group of experienced physicians who care for children with PIUO. In study 2, we tested the feasibility of a standardized approach to the initial workup and interventions for PIUO.

\section{Study 1}

Six physicians, all of whom routinely treat children with pain and SNI at the same medical centre, were asked to participate in the present study. These physicians had between four and 20 years of experience. Training backgrounds included fellowships in general pediatrics, developmental pediatrics, pediatric pain medicine and palliative medicine. The study was approved by the appropriate program and university ethics review (Research Ethics Board of the Child and Family Research Institute and Research Committee of Canuck Place Children's Hospice, Vancouver, British Columbia).

At the outset, a case vignette describing a child with neurological impairment and unexplained pain/irritability despite an extensive workup (Appendix 1) was presented individually to the clinicians; they were then asked to select the medications they would prescribe to treat the PIUO case, and to specify the order in which the medications should be introduced (14). Within the case vignette, the patient had already been treated with acetaminophen, a nonsteroidal anti-inflammatory drug, and baclofen, without effect. Otherwise, there were no prompts or restrictions with regard to which medications the physicians could select or to the order in which they were used. They were instructed to assume that drug dosage and frequency were based on standard formulary references, and were not the subject of the present study.

\section{Study 2}

Children with SNI were recruited either directly from the investigators' clinical programs or from community pediatrics practices. Inclusion criteria were any infant, child or youth with a rare disease and severe neurological impairment experiencing PIUO that was not responding to treatment. These patients were given the opportunity to be followed in a pilot clinic by a physician and nurse for a six-month period. Basic demographic information was obtained for each patient, and health records were reviewed. The study nurse followed the patients throughout the trial and was in frequent contact with families by telephone and e-mail on an as-needed basis, but at a minimum of once per month.

An algorithm was used to diagnose and evaluate pain based on the current literature and expert opinion (Figure 1) (11). The initial step in the algorithm was a history, physical examination and, when possible, detailed observation by diary or video from the family. The information gained from this step guided directed testing via imaging or laboratory studies; if a treatable cause of pain from the history, physical examination or directed studies was found, then it was addressed.

A series of screening tests were undertaken when the initial steps were unrevealing. The screens included urinalysis, gastric $\mathrm{pH}$ testing (if the patient was tube-fed) and abdominal ultrasound. Urinalysis was undertaken to evaluate occult infection or stones as a source of discomfort. Gastric $\mathrm{pH}$ testing was performed to investigate whether acid reflux may be a cause of pain despite the use of antacid therapy. Abdominal ultrasound was recommended in consultation with radiologists as a test to assess for masses and fluid collections; while acknowledged to be low-yield, the low cost and noninvasive nature of this imaging approach supported its inclusion in the present pilot form of an algorithm. Selection of the screening investigations was based, in part, on the expert opinion literature, on the authors' clinical experience and on an evaluation of costs and invasiveness of tests. If an investigation (eg, an imaging study) had been recently undertaken before attendance at the study clinic, it was not repeated; however, the results were recorded in a data sheet. Investigations were repeated if they had not been undertaken since the most recent onset of a pain/ irritability episode (usually within the previous six weeks). The exception was a history and physical examination, which were always performed at the first study clinic visit. If a positive finding was found during the directed or screening phases, follow-up was coordinated with the referring physician or clinical program.

If the history, physical examination, and directed and screening tests did not indicate further evaluation, a standardized medication trial was started. The medication sequence was based on the findings in study 1 . A weighting of the consensus ranking for the indicated drugs was used, and safety profiles and costs were then evaluated with the assistance of a clinical pharmacologist. Starting dose, drug duration and maximal doses to be trialed were also developed by the study pharmacologist. If treatment with a drug had already been attempted with a patient before enrollment in the study, it was not repeated, but the outcome was recorded. Trial duration for any medication was based on knowledge of the specific pharmacokinetics and pharmacodynamics $(15,16)$.

Parents were educated and then asked to complete a painscoring tool designed specifically for nonverbal children, the NonCommunicating Children's Pain Checklist (NCCPC) during the trial. The NCCPC is a valid, reliable tool to assess pain in this population (2). Parents were asked to complete a NCCPC weekly, as well as at any time there were significant changes in the child's condition 


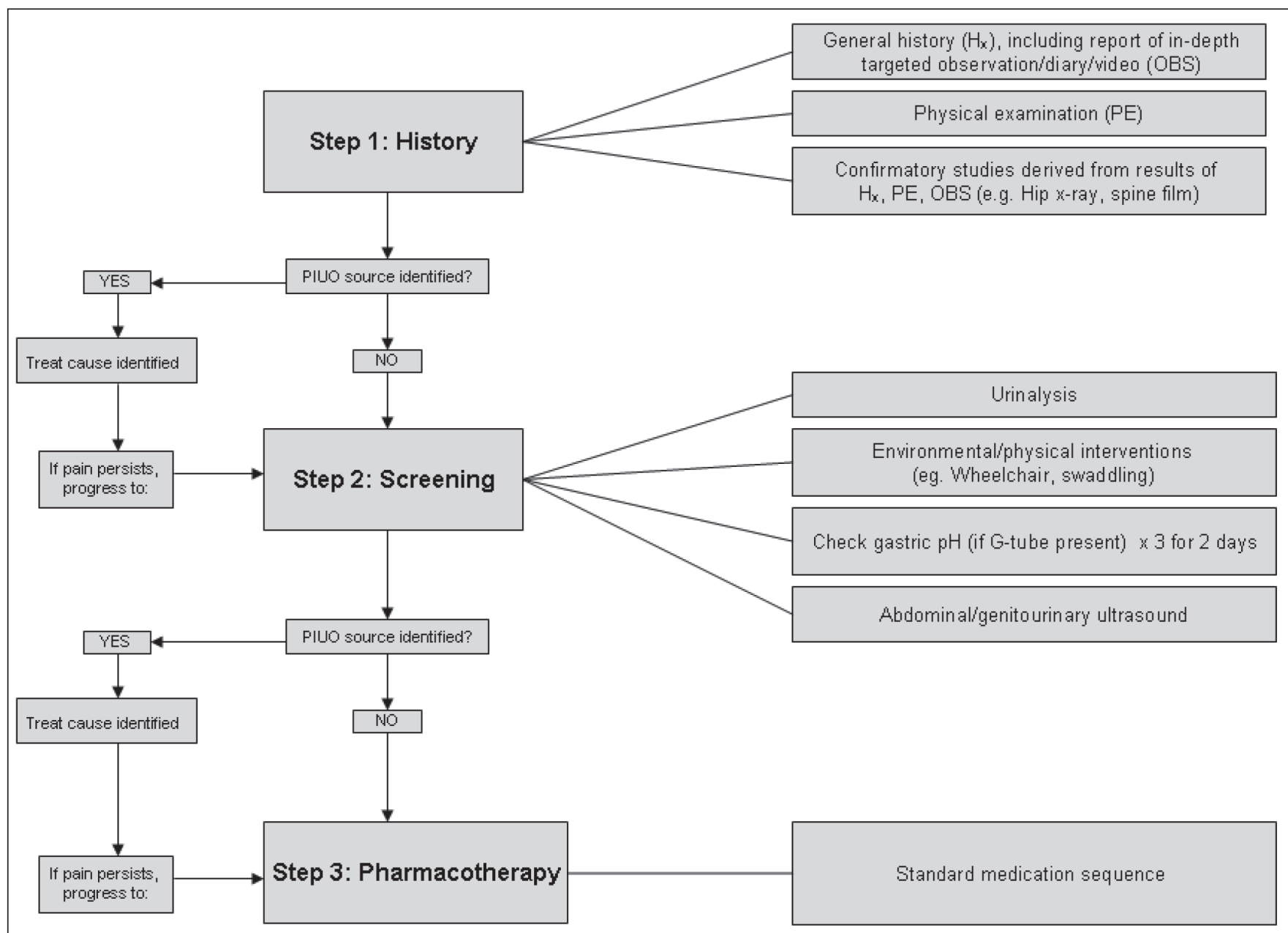

Figure 1) Pathway for unknown pain. G-tube Gastrostomy tube; PIUO Pain and irritability of unknown origin

TABLE 1

Physician drug sequence response to case vignette

\begin{tabular}{llllll}
\hline Physician & First drug & Second drug & Third drug & Fourth drug & Fifth drug \\
\hline A1 & Opioid & Benzodiazepene & Methadone & Gabapentin & Tricyclic antidepressant \\
A2 & Opioid & Gabapentin & Tricyclic antidepressant & $\begin{array}{c}\text { Selective serotinin } \\
\text { reuptake inhibitor }\end{array}$ & Atypical antipsychotic \\
& Opioid & Tricyclic antidepressant & Benzodiazepene & Atypical antipsychotic & Methadone \\
A3 & Atypical antipsychotic & Gabapentin & Benzodiazepene & Tramadol & Opioid \\
B & Gabapentin & Tricyclic antidepressant & Atypical antipsychotic & Benzodiazepene & Tramadol \\
C & Gabapentin & Opioid & Atypical antipsychotic & Benzodiazepene & Methadone \\
D & Benzodiazepene & Gabapentin & Atypical antipsychotic & Opioid & Opioid \\
E & Benzodiazepene & Opioid & Atypical antipsychotic & Methadone & Gabapentin \\
F & & & & & \\
\hline
\end{tabular}

according to their own experience. Parent comments regarding their experience with the project from clinic interactions, telephone calls and e-mails were also collected. The families were able to contact the study physicians and nurse for ongoing evaluation and treatment regarding pain issues for the nine-month period of time that the study was funded. After that, they were rereferred to their referring community clinician.

Study 1

\section{RESULTS}

The results are listed in Table 1, with the physicians identified by letter. One of the physicians provided three different sequences in response to the case vignette. This series is presented in Table 1 as Physician A1, $\mathrm{A} 2$ and $\mathrm{A} 3$. The drug class was reported rather than individual drugs for opioids and benzodiazepines because the within-class differences are medication onset and duration rather than mechanism of action. Tramadol and methadone are reported separately because they have mechanisms of action beyond those of pure opioids.

The results show that eight different medication classes were chosen to treat PIUO. There was some agreement about the three drug classes chosen most often (gabapentinoids, opioids and benzodiazepines). There was no consensus with regard to the sequence for trialing these drugs. Gabapentin was chosen most often as the first (two physicians) or second choice drug (three physicians); opioids were chosen as the first choice medication by three physicians. Benzodiazepines were a first choice for two physicians, but for two other physicians they were the fourth drug to be tried in a sequence. Similarly, one physician started treatment with an atypical antipsychotic (risperidone), but four others placed this drug class as third- or fourth-line drugs. Three other medications appeared as second-, third- and fourth-line drugs: 
TABLE 2

Patient characteristics, number of health care teams involved and results of clinical intervention

\begin{tabular}{|c|c|c|c|c|c|c|c|c|}
\hline Patient & 1 & 2 & 3 & 4 & 5 & 6 & 7 & 8 \\
\hline Age, years & 5 & 12 & 10 & 10 & 14 & 10 & 6 & 17 \\
\hline Sex & Female & Male & Female & Female & Female & Male & Female & Male \\
\hline Pain descriptors & $\begin{array}{l}\text { Self-injury, } \\
\text { disturbed } \\
\text { sleep, } \\
\text { screaming, } \\
\text { arching }\end{array}$ & $\begin{array}{l}\text { Self-injury, } \\
\text { hitting, crying, } \\
\text { irritable }\end{array}$ & $\begin{array}{l}\text { Moaning, } \\
\text { crying, } \\
\text { withdrawal, } \\
\text { flinching }\end{array}$ & $\begin{array}{l}\text { Inconsolable, } \\
\text { cannot lie on } \\
\text { left side, sleep } \\
\text { disturbed }\end{array}$ & $\begin{array}{l}\text { Inconsolable, } \\
\text { disturbed sleep, } \\
\text { self-injury, painful } \\
\text { defecation }\end{array}$ & $\begin{array}{l}\text { Arching, } \\
\text { screaming, } \\
\text { inconsolable }\end{array}$ & $\begin{array}{l}\text { Anticipatory } \\
\text { procedural } \\
\text { pain }\end{array}$ & $\begin{array}{l}\text { Self-injury, } \\
\text { moaning, } \\
\text { withdrawal }\end{array}$ \\
\hline Diagnosis & $\begin{array}{l}\text { Agenesis of } \\
\text { corpus } \\
\text { callosum }\end{array}$ & $\begin{array}{l}\text { CHARGE } \\
\text { syndrome, } \\
\text { autism }\end{array}$ & $\begin{array}{l}\text { Chromosome } \\
13-\mathrm{X} \\
\text { translocation }\end{array}$ & $\begin{array}{l}\text { Ischemic brain } \\
\text { injury with } \\
\text { severe cerebral } \\
\text { palsy }\end{array}$ & $\begin{array}{l}\text { Chromosome 1Xp } \\
\text { duplication, } \\
\text { CHARGE } \\
\text { syndrome }\end{array}$ & $\begin{array}{l}\text { Joubert } \\
\text { syndrome }\end{array}$ & Schizencephaly & $\begin{array}{l}\text { Partial } \\
\text { trisomy } 8 \\
\text { condition }\end{array}$ \\
\hline Number of teams & 14 & 9 & 10 & 7 & 6 & 13 & 6 & 6 \\
\hline $\begin{array}{l}\text { Completed } \\
\text { standardized } \\
\text { workup during study }\end{array}$ & Yes & Yes & No & Yes & Yes & Yes & No & Yes \\
\hline $\begin{array}{l}\text { Drugs given during } \\
\text { study }\end{array}$ & 0 & 0 & 0 & Gabapentin & Methadone & 0 & 0 & Gabapentin \\
\hline Clinical improvement & $\begin{array}{l}\text { No; possible } \\
\text { behaviour } \\
\text { issue }\end{array}$ & $\begin{array}{l}\text { No; possible } \\
\text { behaviour } \\
\text { issue }\end{array}$ & No & Yes & Yes & Yes & $\begin{array}{l}\text { N/A -surgery } \\
\text { upcoming }\end{array}$ & Yes \\
\hline $\begin{array}{l}\text { Change in NCCPC } \\
\text { scores (peak:final) }\end{array}$ & No data & $-20(50: 30)$ & +22 (39:61) & $-42(46: 4)$ & $-51(58: 7)$ & $-44(52: 8)$ & No data & $-3(6: 3)$ \\
\hline
\end{tabular}

N/A Not applicable; NCCPC NonCommunicating Children's Pain Checklist

tricyclic antidepressants, tramadol and methadone. One physician added selective serotonin reuptake inhibitors to the sequence.

\section{Study 2}

Ten children with PIUO referred from clinical sources participated in the pilot study. One chose not to enter the study, and one was found not to have a pain problem at the initial evaluation, leaving eight participants (five female and three male) (Table 2). All were nonverbal and had a variety of SNIs; one was ambulatory, but all required assistance for daily living. Pain-like and irritability behaviours included constant crying, grimacing, a furrowed facial expression, arching and tears. Two of the children had self-injurious behaviour requiring the wearing of protective devices. Disorders of initiating and maintaining sleep were prominent problems.

At entry to the study, the teams already involved in caring for the child and in assessing the child's pain were identified; the number ranged from six to 14 health care teams in addition to their primary care provider (Table 2 ). Three of the families were using alternative medicine approaches including naturopathy or chiropractic treatments. None of them had completed the recommended full workup before study referral as defined in the study protocol with a history and physical examination, targeted investigations or screening studies.

The study physicians were able to stay 'on pathway' with a sequence of history and physical examination, directed and screening tests, and a consensus drug sequence as shown in Table 2. Three patients improved with care initiated during early steps in the algorithm. Only three patients needed to proceed as far as the drug trial sequence while on study. Two were started on gabapentin, the first step in the pilot drug sequence. One patient had received gabapentin before enrollment and treatment was, therefore, initiated with the next drug, an opioid. In two cases the protocol was not completed: in one case due to family reluctance, and in the other because a rapid solution to the problem was identified using the pathway approach.

For five of the eight patients, a decrease in pain-like and irritability behaviours were documented by parents/caregivers using NCCPC scores (Table 2). Scores shown in the table are the peak score and the score at study completion. The peak NCCPC score was the same as the study entry score for all but one patient. The final score was the same as the lowest NCCPC score for all patients. Such reduced pain-like and irritability behaviours were attributed to attention to what were believed to be pain stimuli and modulators, identification of nociceptive sources, use of medications and modification of care settings as identified by the standardized approach.

In four cases, behavioural/environmental issues appeared to occur in concert with an underlying pain due to nociceptive, visceral or neuropathic causes. For at least one of the patients, there appeared to be a significant behavioural/environmental component to their pain, based on serial observations and comparison across observers and settings and reflected in the discrepant NCCPC scores from the parent and school personnel. Whether this arose from differences in perception of the behaviour, responses to setting-specific activities (eg, prolonged time in a wheelchair at school but not at home) or the child's response to different caregivers could not be determined.

\section{DISCUSSION}

PIUO in nonverbal children with SNI poses a significant clinical challenge. The multifactorial nature of the symptoms in the context of a child with multiple health conditions makes both clinical care and research challenging. Initial steps have been the establishment of valid measurement tools that enable research to proceed (17-19); however, numerous and painful investigations are still used in pursuit of an identifiable cause for their symptoms. During this time, the child and family continue to experience distress without an explanation or relief. In an optimal approach, physicians would efficiently treat or rule out identifiable sources of nociceptive-inflammatory pain before moving on to an empirical trial of medications that address purported disruptions in the central nervous system. In the present study, we report the pilot use of a standard pathway (pathway for unknown pain) to establish a baseline approach to managing pain-like behaviours when typical signs and symptoms are difficult to interpret.

The first part of the present study indicates that, even among a group of experienced clinicians, there is no consensus regarding pharmacotherapy to address PIUO. While the literature may suggest that gabapentin is a first-line choice, four of six physicians in our study chose another drug. Furthermore, little evidence supports consistent second-, third-and fourth-line choices, even in the event of therapeutic failure. Highlighting the confusion, one experienced clinician chose three different sequences in response to the case vignette, emphasizing the degree to which consensus or consistency are lacking. 
The second component of our study was designed to assess the feasibility of a common evaluation pathway with the goal of efficiently identifying readily treatable nociceptive-inflammatory pain sources before embarking on an empirical drug trial. Our results show that patients do not undergo a standardized/systematic workup of the problem, as is revealed by the variation in evaluations before referral to the study clinic. Furthermore, these children were being treated by numerous clinical teams without the guidance of a standard protocol; there was considerable probability that for any individual child, constituent teams were working at their own pathway/workup and treatment plan, often compounding inefficient and unproductive management strategies and placing the child at risk for duplicate tests and procedures. The application of a standardized approach in our pilot yielded improvement in several of these children's conditions. It is, therefore, warranted to further explore the use of a standard approach to evaluation and intervention in PIUO. A standardized approach may also lead to improved efficiency in the evaluation of children with SNI and pain-like behaviour. A standard approach, such as the pathway for unknown pain, may be the starting point for studies using methods such as quality improvement and rapid cycle improvement to detect optimal strategies for workup and treatment.

Psychological factors also play an important role in the origin, expression and treatment of pain and irritability, no less in children with SNI. It is challenging to fully understand the antecedent psychosocial elements that may lead to an expression of pain, irritability or distress in intellectually disabled nonverbal children. Furthermore, research is only beginning to explore how parents assess coping with pain in such children (20). More work needs to be performed to identify behavioural interventions that do not require medication (or could supplement the use of medications) to settle pain and irritability in children (21). Experimental studies undertaken by researchers, as well as investigators exploring with parents what works for their children, are equally important avenues of inquiry.

What may be the reasons for such differences regarding evaluation protocol and medication choice? Pediatric Pain Medicine and Palliative Care are new areas of subspecialization, and the research base is not developed. The types of large, multicentre randomized trials that lend themselves to creating evidence for interventions simply do not exist, nor are they likely to be undertaken. Much of the attention in the fields of child development, neurosciences and pediatrics have focused on better understanding of the genetics, preventions and treatments for these conditions, without full appreciation of the need to directly address symptoms such as pain, dyspnea, feeding intolerance and sleep disorders (Siden H, Gregoire MC, et al, manuscript in preparation). Significant advances have been made in understanding the basic biology of nociceptive-inflammatory pain, but our next challenge is to address the neurobehavioural substrate that underlies irritability without a readily identified explanation. For PIUO in children with neurological impairment, physicians do not have adequate evidence other than their own experience, expert opinion and anecdote to guide them. When no consensus whatsoever exists, as was observed in our study, nonexpert physicians cannot easily provide care in the settings where these children are most often seen, such as general pediatrics clinics. Furthermore, the lack of a common initial sequence means that there is no foundation from which to develop future research trials.

Limitations of the present study include the small number of physicians who participated. Involving more clinicians, perhaps across centres, may have helped to develop a clearer picture of the degrees of possible agreement. However, our study engaged physicians who all practice in the same setting and often share patients, highlighting the variability in approach to this challenging population. Delphi processes are one way to develop a consensus among experts; however, given the almost complete lack of pre-existing evidence regarding drug treatments for PIUO, a Delphi study would simply represent what is known from isolated case reports, anecdotes and experience-derived opinions. A limitation to the clinic phase of the study was the small number of patients; however, the present study was intended to be a pilot project focusing on the process of care and on the feasibility of providing pathway-driven care. A second limitation is that the drug sequence we used was a first approximation of a rational approach based on evaluating the agreement inherent in study 1 with additional information from safety profiles and cost. It was not intended to strictly evaluate the medications individually or in sequence; that will be the subject of further study. Therefore, we cannot draw conclusions regarding the overall efficacy of pain treatment in our sample.

While our studies focus on children with impairments in multiple functional areas and major health challenges, better understanding of PIUO may also apply in other situations with individuals whose primary deficit is communication; therefore, individuals with severe mental handicap, autism and adults with dementia may also benefit from a structured approach.

Areas of future study include furthering our understanding of pain in the presence of an SNI, and looking beyond nociception to the developmental pathways that are inherent to an SNI or even the social conditions that all contribute to complex behaviours in this setting. Another significant area of investigation would be improving our knowledge of how medications work in the setting of pain, especially neuropathic/ central pain. Finally, treatment studies need to be conducted using standard, rigorous methods including retrospective case-control studies, publishable n-of- 1 trials, and continuous quality improvement process studies; all of these can result in better care $(22,23)$.

\section{CONCLUSION}

Our two-phase pilot study describes the high variability in using medications to treat PIUO in a vulnerable population, the benefit of using a standardized approach supported by close contact with families and the strong need for additional studies to develop an evidence base.

ACKNOWLEDGEMENTS: The authors thank Ashley Wilson RN, Shalu Duggal MSc, and Dave Marchand BSc RPh for their assistance in conducting the study. They also thank Salma Dewji BSc in assisting with manuscript preparation. This work was supported, in part, by Canuck Place Children's Hospice and by the Rare Diseases Foundation. This work originated at The University of British Columbia. The authors have no conflicts of interest to declare.

\section{APPRENDIX 1. APPROACH TO TREATING UNKNOWN PAIN AND IRRITABILITY IN INDIVIDUALS WITH SNI}

You are presented with a child with a genetic and/or metabolic and/or neurological condition who is displaying "pain-like" behaviours. The child is nonverbal and cannot localize the pain for you, nor can he/she provide a reliable signal as to the degree of discomfort. The behaviours that are pain-like include vocalizations with crying out, grimacing and wrinkling the forehead. There are tears occasionally but not always. There is arching and stiffening, especially of the extensor muscles. The episodes are not predictable, can last from a few minutes to half an hour, and will disrupt sleep.

Having initiated and completed an extensive workup you are not able to determine an obvious or treatable nociceptive/inflammatory cause of the pain-like behaviour. The work up includes a careful history which reveals no specific pattern. The physical examination does not identify a cause; the child has many handicaps but none of these have changed recently. Spasticity and hypertonicity are present but not increased and the child is on baclofen. The seating system has been reviewed. Gastrostomy feeding is tolerated. Constipation is treated. A urinalysis is normal. X-rays of the hips and spine are unchanged. An abdominal ultrasound is not revealing. Treatment with acetaminophen, ibuprofen, positioning and cuddling has not made a difference.

You are left to consider either a nociceptive/inflammatory cause for this pain which you cannot identify, or with a case of CNS "irritability" (which may also be called neuropathic pain, central pain, or neuro-irritability. 
You decide at this point in time that an empirical trial of medication is warranted. There are many classes of medication to choose from - all of them have central nervous system effects, but only some are traditional analgesics, while others are considered "adjuvants" for pain control and span a number of classes.

In this exercise you will choose five medications you include in your trial, and a sequence for the trial.

Assume the following:

- The workup for nociceptive/inflammatory causes (and identifiable/ treatable causes) of the pain-like behaviour is complete.

- You may choose any medication that you wish, but not the three already used (acetaminophen, ibuprofen, baclofen)

- Should you want to trial an anticonvulsant(s), you may choose any one(s) that you wish for the purposes of this exercise. ie, do not assume that the child is already on a particular anti-convulsant (or any at all).

- Do not worry about doses; assume that the "right" dose will be used for the purposes of the exercise. Similarly, do not be concerned about duration of action issues if there are long-and short-acting forms within the same drug class.

\section{REFERENCES}

1. Oberlander TF, Symons F, Van Dongen K, Abu-Saad HH. Pain in individuals with developmental disabilities: Challenges for the future. Prog Pain Res Manag 2003;24:705-24.

2. Breau LM. Non-communicating children's pain checklist: Better pain assessment for severely disabled children. Expert Rev Pharmacoeconomics Outcomes Res 2003;3:327-39.

3. Camfield C, Breau LM, McGrath PJ, Rosmus C, Finley GA. Measuring pain accurately in cognitively impaired children: Validation of a caregiver scale. Ann Neurol 2000;48:544.

4. Stevens B, McGrath P, Yamada J, et al. Identification of pain indicators for infants at risk for neurological impairment: A Delphi consensus study. BMC Pediatrics 2006;6:1-25.

5. Hunt A, Mastroyannopoulou K, Goldman A, Seers K. Not knowing - the problem of pain in children with severe neurological impairment. Int J Nurs Stud 2003;40:171-83.

6. Hunt A, Burne R. Medical and nursing problems of children with neurodegenerative disease. Palliat Med 1995;9:19-26.

7. Lenton S, Stallard P, Lewis M, Mastroyannopoulou K. Prevalence and morbidity associated with non-malignant, life-threatening conditions in childhood. Child Care Health Dev 2001;27:389-98.

8. Houlihan CM, O'Donnell M, Conaway M, Stevenson RD. Bodily pain and health-related quality of life in children with cerebral palsy. Dev Med Child Neurol 2004;46:305-10.

9. Hauer JM. Treatment of persistent pain and irritability in children with neurological impairment. Neuropädiatrie in Klinik und Praxis 2011;10:7-14

10. Oberlander TF, O'Donnell ME, Montgomery CJ. Pain in children with significant neurological impairment. J Dev Behav Pediatr 1999;20:235-43.

11. Siden H, Oberlander TF. Pain Management for children with a developmental disability in a primary care setting. In: Walco GA, Goldschneider KR, eds. Pain in Children: A Practical Guide for Primary Care New Jersey: Humana Press; 2008:29.

12. Gilles EE. "Neuro-irritability" in children with developmental disabilities: The role of autonomic nervous system dysregulation. Neuropädiatrie in Klinik und Praxis 2011;10:15-19.
- Assume you will follow your usual practice. There are issues of medication availability, cost, time needed to assess effect, side-effect profile, etc. You are only required to consider these issues if it is consistent with your usual practice when prescribing these medications. For example, some medications take a few weeks to demonstrate their effect; if it is your usual practice to take this factor into account when prescribing in the kind of scenario described, then do so. Alternatively, if you do not routinely take this into account in your actual practice, then do not do so for the trial.

- Assume that for some drugs there are class equivalents. For example, in the pure opiate group morphine is equivalent to hydromorphone and to fentanyl, so choosing two of these in a sequence would not make sense. On the other hand methadone is not considered a pure opiate, and one may choose this drug in addition to a pure opiate because the mechanism of action will be different.

- Assume you will be using one drug at a time, rather than additively, and assessing for effect

List the top five medications you would use in this situation, and list the order in which you will trial them (assuming that the first one does not work and you need to move to the second, etc)

13. Hauer JM, Wical BS, Charnas L. Gabapentin successfully manages chronic unexplained irritability in children with severe neurologic impairment. Pediatrics 2007;119:e519-22.

14. Bachmann LM, Muhleisen A, Bock A, ter Riet G, Held U, Kessels AG. Vignette studies of medical choice and judgement to study caregivers' medical decision behaviour: Systematic review. BMC Med Res Methodol 2008;8:50.

15. Gray J. Therapeutic Choices, 5th edn. Ottawa: Canadian Pharmacists Association, 2007.

16. Canavero S, Bonicalzi V. Central Pain Syndrome: Pathophysiology, Diagnosis and Management, 1st edn. New York: Cambridge University Press, 2007.

17. Hunt A, Wisbeach A, Seers K, et al. Development of the paediatric pain profile: Role of video analysis and saliva cortisol in validating a tool to assess pain in children with severe neurological disability. J Pain Symptom Manage 2007;33:276-89.

18. Malviya S, Voepel-Lewis T, Burke C, Merkel S, Tait AR. The revised FLACC observational pain tool: Improved reliability and validity for pain assessment in children with cognitive impairment. Paediatr Anaesth 2006;16:258-65.

19. Voepel-Lewis T, Malviya S, Tait AR. Validity of parent ratings as proxy measures of pain in children with cognitive impairment. Pain Manag Nurs 2005;6:168-74.

20. Burkitt CC, Breau LM, Zabalia M. Parental assessment of pain coping in individuals with intellectual and developmental disabilities. Res Dev Disabil 2011;32:1564-71.

21. Slifer KJ, Hankinson JC, Zettler MA, et al. Distraction, exposure therapy, counterconditioning, and topical anesthetic for acute pain management during needle sticks in children with intellectual and developmental disabilities. Clin Pediatr (Phila) 2011;50:688-97.

22. Grimm RH Jr, Shimoni K, Harlan WR Jr, Estes EH Jr. Evaluation of patient-care protocol use by various providers. N Engl J Med 1975;292:507-11.

23. Morris AH. Tools for rigorous experiments in the usual clinical environment. Crit Care Med 2007;35:1776-7. 


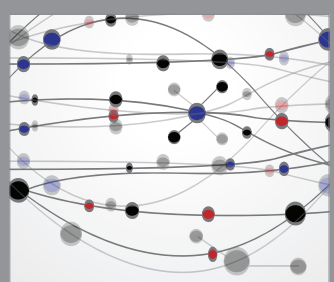

The Scientific World Journal
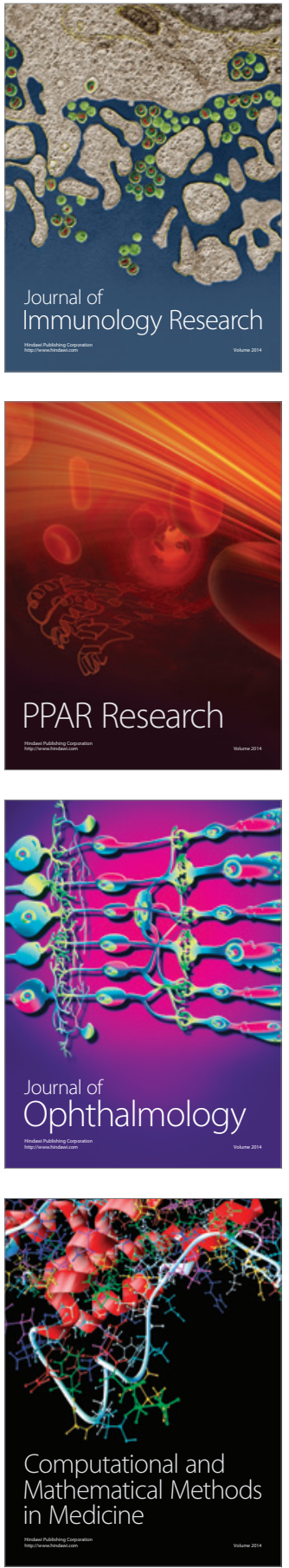

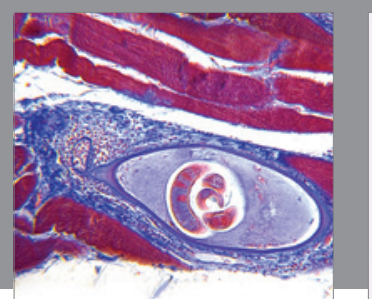

Gastroenterology Research and Practice

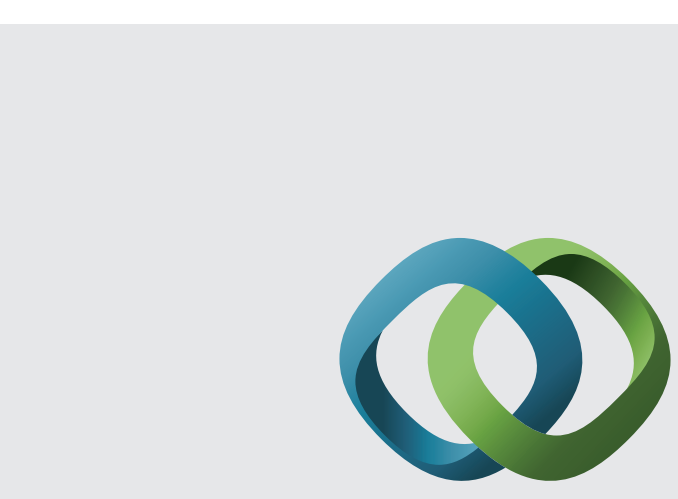

\section{Hindawi}

Submit your manuscripts at

http://www.hindawi.com
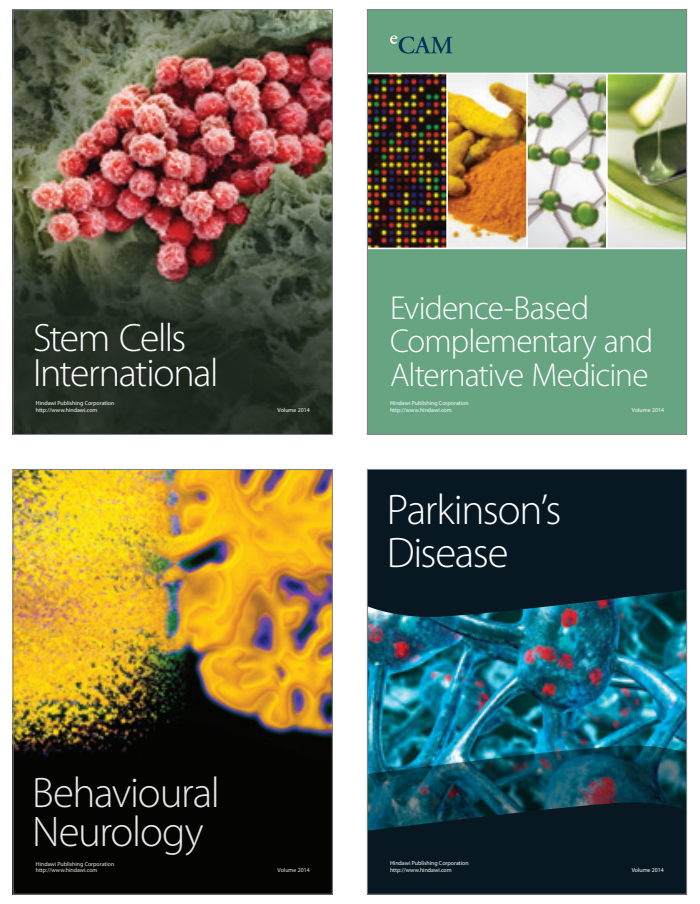
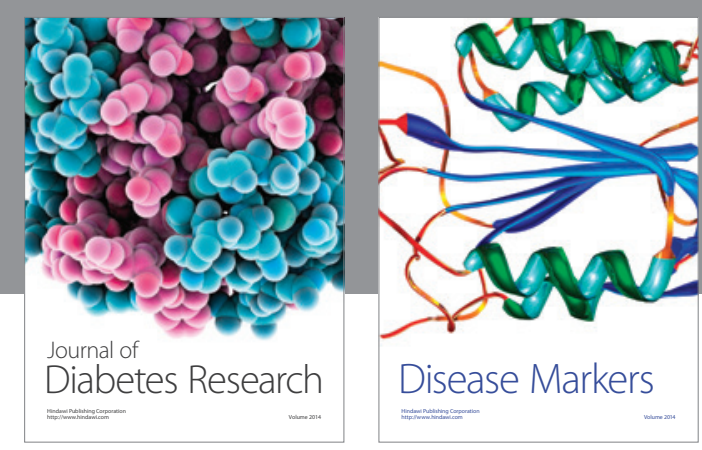

Disease Markers
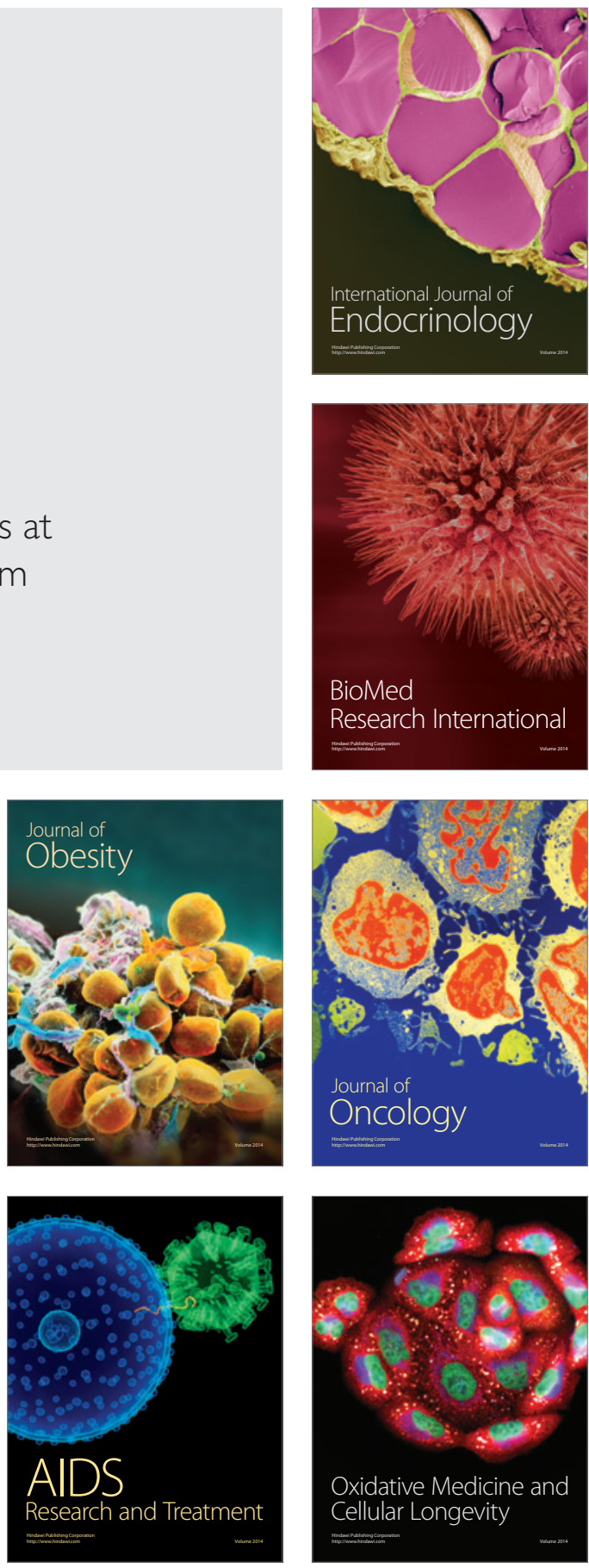